\title{
Parents' Descriptions of Neonatal Palliation as a Treatment Option Prior to Periviable Delivery
}

Shannon Jager ${ }^{1}$, Brownsyne Tucker Edmonds, MD, MPH, MS², Shelley Hoffman, $\mathrm{MPH}^{2}$, Erin Jeffries, MD, $\mathrm{MS}^{2}$, Tatiana Laitano, $\mathrm{MD}^{3}$ and Karen Kavanaugh, $\mathrm{PhD}$, $\mathrm{RN}, \mathrm{FAAN}^{4}$

${ }^{1}$ Indiana University School of Medicine, Indianapolis, IN, ${ }^{2}$ Indiana University School of Medicine, Department of OB/GYN, Indianapolis, IN, ${ }^{3}$ Wayne State University College of Nursing, Detroit, MI

Background and Hypothesis: During periviable deliveries, parents are confronted with overwhelming and challenging decisions, about which they may know little. This study aimed to explore qualitatively the language that mothers and important others (IOs) utilize when discussing palliation, or 'comfort care,' as a treatment option in the context of periviability.

Project Methods: We prospectively recruited pregnant women (and designated IOs) admitted to labor and delivery for a threatened periviable delivery (22-25 weeks GA) at two hospitals between September 2016 and January 2018. Using a semi-structured interview guide, we explored participants' perceptions of palliation and neonatal treatment options. Women were asked items such as, "How was the choice of resuscitation presented to you?" and "What were the options presented?" Research assistants developed a codebook for the interview transcripts, and NVivo 12 was used for qualitative analysis.

Results: Thirty women and 16 IOs were recruited in total. Participants' descriptions of palliation fell into five broad categories - 'doing nothing,' 'not resuscitating,' 'withdrawal of care,' 'implicit comfort care,' and 'explicit comfort care.' The majority of parents perceived comfort care not as a distinct treatment option, but rather as the absence of treatment. Several barriers to the comprehension of comfort care were observed, including subjects' unfamiliarity with the terminology or the inability to remember its explanation during antenatal consultation. Parents described comfort care with either neutral or negative connotations, and even those parents able to discuss the concept neutrally chose resuscitation as their preferred treatment option.

Conclusion and Potential Impact: This study revealed that many parents facing periviable delivery may lack understanding of comfort care as a neonatal treatment option. These parental perceptions highlight the need to improve counseling efforts in order to maximize parents' informed decision-making. 